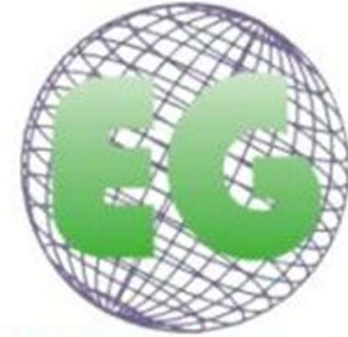

ISSN 1695-6141 $N^{\circ} 60$

\title{
Calidad de las compresiones torácicas en resucitación cardiopulmonar practicada en condiciones extremas de temperatura ambiental
}

Quality of chest compressions in cardiopulmonary resuscitation performed in extreme ambient temperature conditions

\author{
Maria Luisa Fernández-González-de-la-Riva ${ }^{1}$ \\ Manuel Piñero-Zapata ${ }^{1}$ \\ Maria Luisa González-de-la-Riva-Troncoso² \\ 1 Universidad Católica San Antonio de Murcia. España. marisa.fernandez.gon@gmail.com \\ ${ }^{2}$ Hospital Comarcal de Vinaroz. Castellón. España.
}

\section{https://doi.org/10.6018/eglobal.406401}

Recibido: $11 / 12 / 2019$

Aceptado: 12/04/2020

\section{RESUMEN:}

En la atención del equipo de profesionales de la salud en una parada cardiorrespiratoria extrahospitalaria (PCREH), se pueden encontrar en condiciones extremas de temperatura ambiental, según la zona donde se encuentren, la época del año, e incluso la franja horaria.

Objetivo: El objetivo de este trabajo fue comprobar si la eficacia de la Reanimación Cardiopulmonar $(\mathrm{RCP})$ se veía afectada en ambientes de temperatura calor $\left(40^{\circ} \mathrm{C}\right)$ y frío $\left(0^{\circ} \mathrm{C}\right)$ extremos, en comparación con una RCP en temperatura ambiental $\left(22^{\circ} \mathrm{C}\right)$.

Método: Se realizaron 2 minutos de compresiones torácicas (CT) ininterrumpidas en las condiciones de temperatura de $40^{\circ} \mathrm{C}, 0^{\circ} \mathrm{C}$ y $22^{\circ} \mathrm{C}$, en un maniquí realista Resusci Anne QCPR con SIMPAD.

Resultados: 30 participantes, tanto con formación previa en RCP $(86,7 \%)$ o sin ella $(13,3 \%)$, formaron parte de la muestra, 26 mujeres $(86,7 \%)$ y 4 hombres $(13,3 \%)$, con una media de edad de $27,37 \pm 8,88$ años. No se encontraron diferencias estadísticamente significativas en la eficacia de la RCP; pero sí que hubo diferencias entre el número total de CT $(p=0,042)$, la profundidad media de las CT $(p=0,015)$ y en la frecuencia media $(p=0,034)$. La escala de Borg de esfuerzo percibido (RPE) mostró una media de $4,4 \pm 1,303$ a $22^{\circ} \mathrm{C}, 4,97 \pm 1,402$ a $40^{\circ} \mathrm{C}$ y $4,87 \pm 2,08$ a $0^{\circ} \mathrm{C}(p>0,05)$. No hubo diferencias significativas en el lactato capilar.

Conclusiones: Se concluye que no existen diferencias significativas entre las tres condiciones, aunque parece que la temperatura ambiental y la fría se asemejan en los resultados y la temperatura calor ofrece una ligera desventaja al respecto.

Palabras clave: Reanimación cardiopulmonar, reanimador, fatiga, compresiones torácicas, temperatura ambiental, simulación.

\section{ABSTRACT:}

Under the care of a team of health professionals in an out-of-hospital cardiorespiratory arrest (PCREH), they can be found in conditions in extreme conditions of ambient temperature, depending on the area where they are located, the time of the year, and even the time of day. 
Objective: The objective of this study was to verify whether the efficacy of Cardiopulmonary Resuscitation (CPR) was affected by extreme heat $\left(40^{\circ} \mathrm{C}\right)$ and cold $\left(0^{\circ} \mathrm{C}\right)$ temperature environments, compared to a CPR in ambient temperature $\left(22^{\circ} \mathrm{C}\right)$.

Method: 2 minutes of uninterrupted external chest compressions (ECC) were performed under ambient temperature conditions of $40^{\circ} \mathrm{C}, 0^{\circ} \mathrm{C}$ and $22^{\circ} \mathrm{C}$, in a realistic mannequin Resusci Anne QCPR with SIMPAD.

Results: 30 participants, both people with prior training in CPR $(86.7 \%)$ and without it $(13.3 \%)$, were part of the sample, 26 women (86.7\%) and 4 men (13.3\%), with a mean age of $27.37 \pm 8.88$ years. No statistically significant differences were found in the efficacy of CPR; but there were differences between the total number of CT $(p=0.042)$, the average depth of the ECC $(p=0.015)$ and the average frequency $(p=0.034)$. The Borg scale of perceived exertion (RPE) showed an average of $4.4 \pm 1.303$ at $22^{\circ} \mathrm{C}, 4.97 \pm 1.402$ at $40^{\circ} \mathrm{C}$ and $4.87 \pm 2.08$ at $0^{\circ} \mathrm{C}(\mathrm{p}>0.05)$. There were no significant differences in hair lactate.

Conclusions: It is concluded that there are no significant differences between the three conditions, although it seems that the cold and room temperatures resemble results and that the heat temperature offers a slight disadvantage of the others.

Key words: cardiopulmonary resuscitation, resuscitator, fatigue, chest compressions, ambient temperature, simulation.

\section{INTRODUCCIÓN}

La reanimación cardiopulmonar $(\mathrm{RCP})$ básica está compuesta por compresiones torácicas (CT) o cardíacas de calidad y ventilaciones asistidas, incluyéndose en ella el uso del desfibrilador. Existen 5 componentes de una RCP de alta calidad como la fracción de compresión torácica ( $\mathrm{FCT}$ ), frecuencia de $\mathrm{CT}$, profundidad de $\mathrm{CT}$, expansión torácica o compresión residual y ventilación ${ }^{(1)}$. Concretamente, la profundidad será de aproximadamente $5-6 \mathrm{~cm}$ en el adulto y la frecuencia $>100 \mathrm{cpm}$, así como permitir que el tórax se reexpanda después de cada compresión (2).

Si entendemos las compresiones torácicas como una actividad física para el reanimador, podemos definirla como intensa y de corta duración debido a que, a partir de los $1 \frac{1}{2}-2$ minutos, la fatiga del reanimador aumenta y la calidad de las compresiones comienza a disminuir significativamente ${ }^{(3-7)}$.

Los sistemas que participan en la producción de energía en el organismo vienen determinados por la intensidad, así pues, siendo una actividad intensa y de corta duración, la predominancia de la vía de reposición de ATPs sería la vía anaeróbica glucolítica(8). $^{(8)}$.

En la PCR extrahospitalaria, los profesionales sanitarios están expuestos a cualquier contexto climatológico. En referencia a la temperatura ambiental, las condiciones de calor y frío extremos afectan de diferente forma al organismo. Metabólicamente, en un contexto caluroso, se ve aumentada la glucogenólisis muscular, la glucolisis y la degradación del fosfato de alta energía durante el ejercicio ${ }^{(9)}$. En actividades repetidas e intensas, cuando se alcanza el estado de hipertermia, el rendimiento físico se ve disminuido; se sugiere que esta reducción está relacionada con un aumento de la temperatura central y no con los agentes metabólicos fatigantes $\operatorname{conocidos}^{(10,11)}$, así como de la disponibilidad de carbohidratos. En cambio, en ambientes fríos se ha observado un aumento de la resistencia, probablemente relacionada con una reducción en la velocidad glucogenolítica(12).

El rendimiento físico en ambientes fríos se ve deteriorado por la disminución de las funciones mecánicas, bioquímicas y neurales del músculo a causa de una disminución de la temperatura de este. En cambio, se ha observado que en 
ambientes calurosos el rendimiento aumenta cuando se realiza una actividad intensa de corta duración. Si esta actividad se repite, la relación positiva se reduce y el rendimiento se deteriora(13).

El objetivo de nuestro estudio fue determinar si existen diferencias en la calidad de las compresiones torácicas en la resucitación cardiopulmonar en función de la temperatura ambiental donde se realice la RCP.

\section{MÉTODO}

Estudio analítico comparativo de la calidad de las compresiones torácicas en la resucitación cardiopulmonar simulada y practicada sobre un maniquí específico para $\mathrm{RCP}$ en diferentes condiciones de temperatura ambiental realizadas en laboratorio en las instalaciones de la Universidad Católica San Antonio de Murcia (UCAM).

Se llevaron a cabo tres pruebas diferentes consistentes en la realización de compresiones torácicas de calidad ininterrumpidamente durante 2 minutos sobre maniquí en el suelo en cada una de las pruebas. Las condiciones de temperatura ambiental de la sala/laboratorio para cada una de las tres pruebas fueron de $22^{\circ}, 40^{\circ}$ y $0^{\circ} \mathrm{C}$ respectivamente. En cada una de las condiciones se realizaba una aclimatación previa a la temperatura ambiental de 5 minutos por parte del participante. Durante la realización de la prueba los sujetos no tenían información sobre la calidad de la prueba ni el tiempo transcurrido. Al finalizar los dos minutos se les preguntaba sobre el grado de esfuerzo percibido de la prueba, según la escala de Borg, y se les medía el lactato en sangre capilar mediante punción en el dedo índice. Si se realizaban las pruebas de dos temperaturas en una misma sesión, los participantes permanecían en reposo fuera de las salas de laboratorio durante quince minutos entre pruebas a fin de conseguir su estado basal nuevamente.

Las tres pruebas del estudio se realizaron durante los meses de abril, mayo, junio y julio de 2019.

El tamaño de la muestra fue de 30 participantes con muestreo de conveniencia, que se presentaban en ausencia de enfermedad en fase aguda y/o no controla, mayores de edad que hubiesen expresado su voluntad de participar en el estudio. Los criterios de exclusión fueron personas con enfermedad en fase aguda, con lesiones osteomusculares de las extremidades superiores, personas que no hubieran cumplido la mayoría de edad y personas que no hubieran firmado el consentimiento informado. Se retiraron los participantes que se ausentaron a una o más jornadas de las pruebas y aquellos que expresaron de forma voluntaria la retirada de su consentimiento para participar en el estudio.

Las compresiones torácicas se realizaron sobre un Maniquí de simulación Resusci Anne $\mathrm{QCPR}^{\circledR}$ con dispositivo operativo de control SimPad ${ }^{\circledR}$ de la empresa Laerdal Medical AS; para la medición del lactato en sangre se utilizó el dispositivo Accutrend Plus de Roche Diagnostics con tiras reactivas BM-Lactate, y para la valoración subjetiva de la dureza de la prueba se hizo uso de la escala validada de esfuerzo percibido de Borg modificada CR-10 (RPE). 
Los datos obtenidos en el SimPad ${ }^{\circledR}$ junto con el resto de datos de las pruebas y de los participantes fueron tabulados y exportados al programa de tratamiento estadístico para su análisis.

El análisis estadístico, se realizó con el programa Statistical Package for the Social Sciences (SPSS). La descripción de las variables se realizó mediante el recuento de frecuencias y sus porcentajes y por sus medias y desviaciones estándar (DE); así como su mediana y amplitud interquatílica, en función de su normalidad. Para determinar la normalidad en las variables cuantitativas se realizó la prueba de Shapiro-Wilk. Las diferencias entre las variables se establecieron por la "ji-cuadrado" o por la "F" de Fisher, así como "t" de Student o "U" de Mann Whitney; si se comparaban más de dos grupos se empleó la prueba de ANOVA o Kruskal-Wallis. En la búsqueda de la asociación dentro de muestras relacionadas, se hizo uso de la prueba "t" de Student o por la prueba de Wilcoxon; si había más de dos el estadístico utilizado fue la prueba de Friedman.

Para las correlaciones bivariadas se utilizó Pearson o Spearman. El nivel de significación fue el de $p<0,05$.

\section{RESULTADOS}

La muestra estuvo formada por 30 participantes de los cuales, 26 eran mujeres $(86,7 \%)$ y 4 hombres $(13,3 \%)$. Las Tablas 1 y 2 definen las características de los individuos de la muestra.

Tabla 1. Características de los individuos de la muestra - Variables categóricas.

\begin{tabular}{ccccc}
\hline Variable & & & \\
Sexo & Mujeres & Hombres & \multicolumn{1}{c}{ Total } \\
& $26(86,7 \%)$ & $4(13,3 \%)$ & $30(100 \%)$ & \\
\hline Clasificación IMC & Infrapeso & Normopeso & Sobrepeso Obesidad \\
\hline Formación previa & $3(10,9 \%)$ & $20(66,7 \%)$ & $5(16,7 \%)$ & $2(6,7 \%)$ \\
& F. Avanzada. & F. Básica & F. Nula \\
\hline
\end{tabular}

IMC = Índice de Masa Corporal .

Tabla 2. Características de los individuos de la muestra - Variables cuantitativas.

\begin{tabular}{cccccc}
\hline & Media & Mediana & DE & AIQ & Mín. - Máx. \\
Edad & 27,37 & $\mathbf{2 5}$ & $\pm 8,880$ & $\mathbf{7}$ & $19-58$ \\
Altura & 167,43 & $\mathbf{1 6 7}$ & $\pm 9,565$ & $\mathbf{9}$ & $154-196$ \\
Peso & $\mathbf{6 3 , 1 6 0}$ & 62,4 & $\mathbf{\pm 1 0 , 9 5 8 3}$ & 12,4 & $47,9-92,7$ \\
IMC & $\mathbf{2 2 , 7 1 2}$ & 22,1 & $\mathbf{\pm 3 , 2 6 7 7}$ & 4 & $18,3-32,1$ \\
\hline
\end{tabular}

$\mathrm{DE}=$ desviación estándar; $\mathrm{AIQ}=$ amplitud intercuartílica.

Se encontraron diferencias significativas en el número total de compresiones torácicas realizadas durante la prueba $(p=0,042)$, en la profundidad media $(p=0,015)$ y en la 
frecuencia media de las mismas $(p=0,034)$ (Tabla 3 ). En todas las condiciones las variables se comportaron con normalidad, a excepción del \% de RCP en $\mathrm{T}^{\mathrm{a}}$ ambiente y Tª fría.

Tabla 3. Resultados de la prueba a partir del maniquí SIMPAD - Variables cuantitativas.

\begin{tabular}{|c|c|c|c|c|c|c|c|c|c|c|}
\hline & \multicolumn{3}{|c|}{$\mathrm{T}^{\mathrm{a}}$ ambiente } & \multicolumn{3}{|c|}{$\mathrm{T}^{\mathrm{a}}$ calor } & \multicolumn{4}{|c|}{$T^{a}$ fría } \\
\hline Variable & $\begin{array}{l}\text { Media } \\
\pm D E\end{array}$ & $\begin{array}{l}\text { Mediana } \\
\text { (AIQ) }\end{array}$ & $\begin{array}{l}\text { Mín. - } \\
\text { Máx. }\end{array}$ & $\begin{array}{l}\text { Media } \\
\pm D E\end{array}$ & $\begin{array}{c}\text { Mediana } \\
\text { (AIQ) }\end{array}$ & $\begin{array}{l}\text { Mín. } \\
\text { - } \\
\text { Máx. }\end{array}$ & $\begin{array}{c}\text { Media } \\
\pm D E\end{array}$ & $\begin{array}{c}\text { Mediana } \\
\text { (AIQ) }\end{array}$ & $\begin{array}{l}\text { Mín. } \\
\text { - } \\
\text { Máx. }\end{array}$ & $\begin{array}{c}p \\
\text { valor }\end{array}$ \\
\hline$\%$ RCP & $\begin{array}{r}83,22 \\
\pm 17,509\end{array}$ & $\begin{array}{r}89 \\
(63)\end{array}$ & $\begin{array}{l}37- \\
100\end{array}$ & $\begin{array}{c}75,27 \\
\pm 20,843\end{array}$ & $75(67)$ & $\begin{array}{l}33- \\
100\end{array}$ & $\begin{array}{l}81,91 \pm \\
18,285\end{array}$ & $90(54)$ & $\begin{array}{c}45- \\
99\end{array}$ & 0,262 \\
\hline $\mathrm{N}^{\circ} \mathrm{CT}$ & $\begin{array}{r}221,37 \\
\pm 20,508\end{array}$ & $\begin{array}{l}220 \\
(24)\end{array}$ & $\begin{array}{c}169- \\
274\end{array}$ & $\begin{array}{r}229,07 \\
\pm 22,706\end{array}$ & $\begin{array}{c}222,50 \\
(25)\end{array}$ & $\begin{array}{c}175- \\
278\end{array}$ & $\begin{array}{c}231 \pm \\
28,928\end{array}$ & $\begin{array}{c}221,50 \\
(45)\end{array}$ & $\begin{array}{c}191- \\
294\end{array}$ & 0,042 \\
\hline $\begin{array}{l}\text { Prof. } \\
\text { med CT }\end{array}$ & $\begin{array}{r}50,53 \\
\pm 6.694\end{array}$ & $\begin{array}{c}50,50 \\
(12)\end{array}$ & $38-63$ & $\begin{array}{r}48,13 \\
\pm 8,332\end{array}$ & $47(30)$ & $\begin{array}{l}33- \\
63\end{array}$ & $\begin{array}{c}47,17 \pm \\
6,919\end{array}$ & $50(27)$ & $\begin{array}{l}35- \\
62\end{array}$ & 0,015 \\
\hline $\begin{array}{c}\text { Frec. } \\
\text { med CT }\end{array}$ & $\begin{array}{r}110,93 \\
\pm 10,544\end{array}$ & $\begin{array}{l}109 \\
(53)\end{array}$ & $\begin{array}{l}85- \\
138\end{array}$ & $\begin{array}{r}115,17 \\
\pm 11,350\end{array}$ & $112(52)$ & $\begin{array}{l}88- \\
140\end{array}$ & $\begin{array}{c}116,3333 \pm \\
14,40626\end{array}$ & $\begin{array}{c}112 \\
(23,5)\end{array}$ & $\begin{array}{l}96- \\
148\end{array}$ & 0,034 \\
\hline
\end{tabular}

$\% \mathrm{RCP}=$ Porcentaje de Reanimación Cardiopulmonar correcta; $\mathrm{N}^{\circ} \mathrm{CT}=$ Número de compresiones torácicas totales realizadas; Prof. med CT = Profundidad media de las compresiones; Frec. med CT = Frecuencia media de las compresiones torácicas; $\mathrm{DE}=$ desviación estándar; $\mathrm{AIQ}=$ rango intercuartílico; Mín. = mínimo; Máx. = máximo.

Las medias de puntuación obtenidas del RPE por los participantes en cada una de las tres pruebas de temperatura fueron las siguientes: 4,4 $\pm 1,303$ en temperatura ambiente, $4,97 \pm 1,402$ en calor y $4,87 \pm 2,08$ en frío $(p>0,05)$. En temperatura calor y fría, se trató como variable normal y en ambiente como no paramétrica.

Se encontraron diferencias estadísticamente significativas $(p<0,05)$, en cuanto a las variables obtenidas a partir del maniquí SIMPAD, entre la "profundidad media de las compresiones torácicas" en temperatura ambiente y temperatura calor $(50,53 \mathrm{~mm}$ vs $48,13 \mathrm{~mm} ; \mathrm{p}=0,046)$, así como en temperatura ambiente y temperatura fría $(50,53 \mathrm{~mm}$ vs $47,17 \mathrm{~mm} ; \mathrm{p}=0,030$ ) (Figura 1 ).

Figura 1. Profundidad media de las compresiones torácicas en las distintas temperaturas $\left(22^{\circ}, 40^{\circ}\right.$ y $\left.0^{\circ}\right)(p<0,05)$.

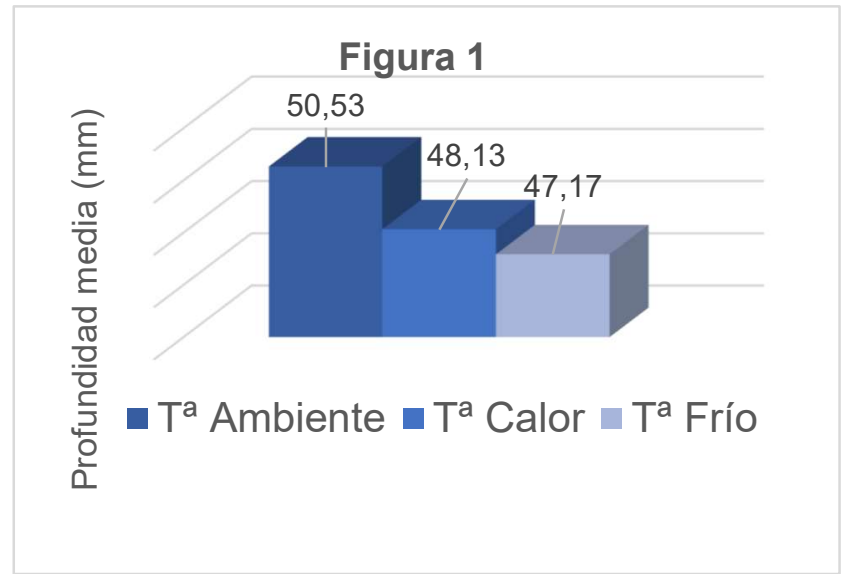

También en relación la "frecuencia media de las compresiones torácicas" en 
temperatura ambiental y calor (110 cpm vs $115 \mathrm{cpm} ; \mathrm{p}=0,025)$ y temperatura ambiental y frío (110 cpm vs $116 \mathrm{cpm} ; \mathrm{p}=0,028)$ (Figura 2).

Figura 2. Frecuencia media de compresiones torácicas en las distintas temperaturas $\left(22^{\circ}, 40^{\circ}\right.$ y $\left.0^{\circ}\right)(p<0.05)$.

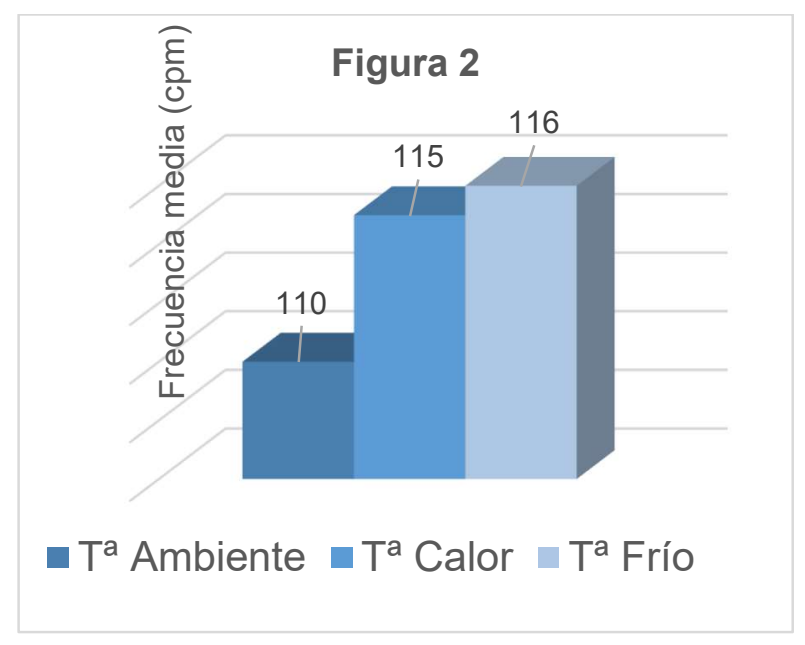

Las diferencias del "número de compresiones totales" entre temperatura ambiental y calor (221ct vs $229 \mathrm{ct} ; \mathrm{p}=0,032$ ); y temperatura ambiental y frío (221ct vs $231 \mathrm{ct}$; $\mathrm{p}=0,046$ ), fue estadísticamente significativa (Figura 3).

Figura 3. Número de compresiones torácicas totales en las distintas temperaturas $\left(22^{\circ}, 40^{\circ}\right.$ y $\left.0^{\circ}\right)(p<0.05)$.

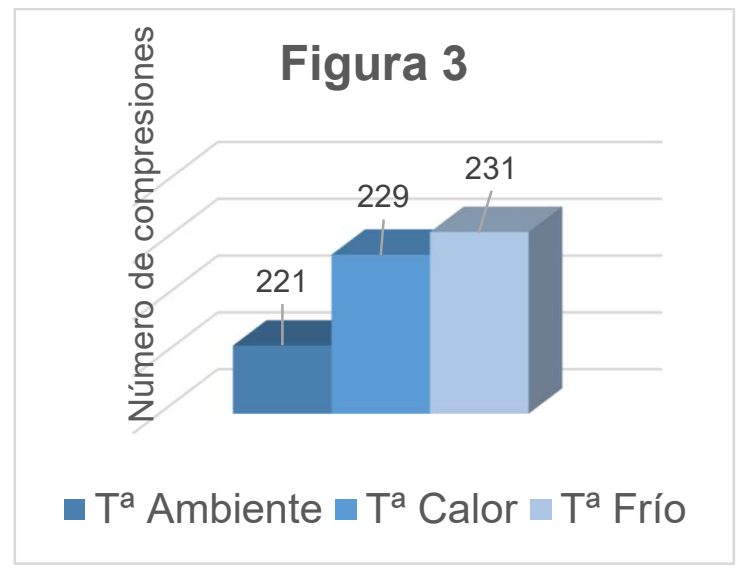

\section{DISCUSIÓN}

Para la discusión de los resultados, se ha hecho uso de estudios en el ámbito de las Ciencias de la Actividad Física, debido a las dificultades para compararlo con otros estudios del mismo enfoque. Así pues, los autores queremos destacar la dificultad de la discusión de este, por lo que serían necesarios más estudios al respecto.

Como resolución del objetivo principal, en la tabla 3, se puede ver que en la variable "\%RCP" no existían diferencias significativas entre las diferentes condiciones; sin embargo, si observamos las variables en las que se basa la RCP, frecuencia, 
profundidad y número de compresiones, sí que existían estas diferencias significativas. De hecho, entre los parámetros que engloban a la RCP, se observa que, respecto a la temperatura ambiente, el número de compresiones aumentaba tanto en la temperatura calor como en la fría al mismo tiempo que la profundidad media disminuía y estas diferencias se reflejaban significativamente. Esto puede ser explicado por el gasto cardíaco, en el que el aumento de las primeras compensaría la disminución de la segunda, no viéndose afectado por tanto y manteniendo la eficacia de la $\operatorname{RCP}^{(14)}$.

Del mismo modo, la muestra en las condiciones de $\mathrm{T}^{\mathrm{a}}$ ambiental y frío, realizaron RCP avanzada, mientras que el restó disminuyó la calidad en la temperatura calor. Se podría explicar, mediante la termorregulación del cuerpo; en la condición de calor, el cuerpo necesitaría de un tiempo superior a la duración de la prueba para realizar la sudoración y así refrigerar el cuerpo y mantener el rendimiento; mientras que, en la condición de frío, la contracción muscular continua favorecería el aumento de la temperatura corporal, y como se observa en el apartado de resultados, existe una correlación directa entre la eficacia de la RCP y el aumento de la temperatura corporal durante la $\operatorname{prueba}^{(15)}$.

La escala de esfuerzo percibido (RPE) nos ofrece una valoración subjetiva del rescatador sobre el esfuerzo físico. En este estudio(16), en el que se realizaron 2 minutos de RCP, el valor medio del RPE fue de $3 \pm 2$; por lo que fue inferior a nuestros resultados, sin embargo, los participantes eran una muestra heterogénea de profesionales de salud entrenados en RCP. Asimismo, se encontraron diferencias significativas respecto al aumento de la intensidad del esfuerzo en temperatura calor. Como se expresa en el artículo, en una actividad de intensidad fija el rendimiento se ve deteriorado, así como una hipertermia inducida por el ejercicio se asocia a una fatiga central(17). Es posible que bajo las recomendaciones del Consejo Europeo de Resucitación (ERC) de intercambiar el resucitador cada 2 minutos, en temperatura calor se pudiera realizar este cambio con anterioridad, para garantizar la eficacia de la $\mathrm{RCP}(18)$.

Consideramos que sería necesario realizar un estudio comparativo que incluyera un número mayor de " $n$ " y con un diseño de mayor duración, en el que los reanimadores se aproximaran a una fatiga extrema y monitorizar a lo largo del tiempo las variables pertinentes, para obtener una información evolutiva más detallada.

\section{Limitaciones del estudio}

A pesar de los esfuerzos por recrear el diseño de estudio, existieron diferentes limitaciones de carácter técnico que pudieron influir en la investigación y tener alguna repercusión en los resultados.

En primer lugar, las pruebas fueron realizadas en laboratorio, no en ámbito real, por lo que no se conoce si se pudieran reproducir todos los aspectos de una reanimación real; existen variables en las que el contexto puede afectar al reanimador; la motivación, el ambiente, el equipo, la toma de decisiones pueden afectar de manera inherente a la calidad de un proceso. Una segunda limitación podría, encontrarse en la ubicación de la prueba en temperatura fría, ya que el espacio para la realización de la prueba era muy limitado, por lo que las dimensiones corporales de algunos participantes quizá pudieron afectar a la realización de las compresiones torácicas. 


\section{CONCLUSIONES}

En conclusión, la calidad global de las compresiones torácicas no ha mostrado diferencias estadísticamente significativas cuando son realizadas en temperaturas ambientales extremas de calor o de frío. Del mismo modo, la mediana que representa el porcentaje de compresiones torácicas realizadas correctamente en calor extremo fue inferior a la encontrada en temperatura ambiental y fría. Ello muestra una tendencia al empeoramiento de la calidad en esa condición de temperatura frente a las otras dos a pesar de no haber encontrado significación estadística. En cuanto al RPE, fue similar en las tres pruebas realizadas, sin embargo, la temperatura de calor extremo parece ser la peor condición para realizar compresiones torácicas, cualitativamente hablando, tomando como referencia la temperatura ambiental a $22^{\circ} \mathrm{C}$.

Finalmente, se han encontrado diferencias estadísticamente significativas en los parámetros involucrados en las compresiones torácicas, la profundidad media, el número de compresiones totales y la frecuencia media de las compresiones torácicas entre las tres pruebas de temperatura; sin embargo, no parecen haber afectado al resultado global de la calidad.

\section{Financiación}

Acuerdo de colaboración con Roche Diagnostics SLU.

\section{REFERENCIAS}

1. Christenson J, Caen AR De, Bhanji F, Abella BS, Kleinman ME, Dana P, et al. Declaración de consenso de la AHA. 2014.

2. Kg M, Resuscitation $\mathrm{E}$, Guidelines C.

Recomanacions_ERC_2015_Principals_novetats.pdf. 2015;1-8. Disponible en: http://www.cercp.org/images/stories/recursos/Documentos/Recomanacions ERC 201

5 Principals novetats.pdf

3. Blas G De, Garc P, Cobo-va C. Electrophysiology of Muscle Fatigue in Cardiopulmonary Resuscitation on Manikin Model. 2015;30-7.

4. Kleinman, M. E., Brennan, E. E., Goldberger, Z. D., Swor, R. A., Terry, M., Bobrow, B. J., Rea, T. (2015). Part 5: Adult Basic Life Support and Cardiopulmonary Resuscitation Quality. Circulation, 132(18 suppl 2), S414 -S435. doi:10.1161/cir.0000000000000259.

5. Sánchez B, Algarte R, Piacentini E, Trenado J, Romay E, Cerdà M, et al. Low compliance with the 2 minutes of uninterrupted chest compressions recommended in the 2010 International Resuscitation Guidelines . J Crit Care [Internet]. 2015;30(4):711-4. Disponible en: http://dx.doi.org/10.1016/j.jcrc.2015.03.001

6. Gianotto-Oliveira R, Gianotto-Oliveira G, Gonzalez MM, Quilici AP, Andrade FP, Vianna CB, et al. Quality of continuous chest compressions performed for one or two minutes. Clinics. 2015;70(3):190-195.

7. Kılıç D, Göksu E, Kılıç T, Buyurgan CS. Resuscitation quality of rotating chest compression providers at one-minute vs. two-minute intervals: A mannequin study. Am J Emerg Med [Internet]. 2018;36(5):829-33. Disponible en: http://dx.doi.org/10.1016/j.ajem.2017.10.035.

8. López J, Almudena F. Fisiología del Ejercicio - López Chicharro.pdf. 2006. p. 987. 
9. Carey MF, Stathis CG, Snow RJ, Febbraio MA, Hargreaves M. Influence of elevated muscle temperature on metabolism during intense, dynamic exercise. Am J Physiol Integr Comp Physiol. 2017;271(5):R1251-5.

10. Nybo L, Rasmussen P, Sawka MN. Performance in the Heat - Physiological Factors of Importance for Hyperthermia-Induced Fatigue. 2014;4(April):657-89.

11. Girard O, Brocherie F, Bishop DJ. Sprint performance under heat stress: A review. 2016;25:79-89.

12. Parkin JM, Carey MF, Zhao S, Febbraio MA. Effect of ambient temperature on human skeletal muscle metabolism during fatiguing submaximal exercise. J Appl Physiol. 2017;86(3):902-8.

13. Racinais S, Oksa J. Temperature and neuromuscular function. Scand J Med Sci Sport. 2010;20(SUPPL. 3):1-18.

14. H. Wilmore, J; L. Costill, D. Control cardiovascular durante el ejercicio. En: H. Wilmore, J; L. Costill, D. Fisiología del esfuerzo y del ejercicio. $6^{a}$ ed. Barcelona: Editorial Paidotribo, 2007. P. 221-241.

15. Vogt J. Enciclopedia de Salud y Seguridad en el Trabajo. 4-5 p.

16. Trenado J, Quintana S, Baltasar S, Fern M, Mestre J. The physiological effect on rescuers of doing 2 min of uninterrupted chest compressions. Res. 2007;74:108-12.

17. Nybo L, Rasmussen P, Sawka MN. Performance in the Heat - Physiological Factors of Importance for Hyperthermia-Induced Fatigue. 2014;4 (April):657-89.

18. Mcdonald $\mathrm{CH}$, Heggie J, Jones $\mathrm{CM}$, Thorne CJ, Hulme J. Rescuer fatigue under the 2010 ERC guidelines, and its effect on cardiopulmonary resuscitation (CPR) performance. Joirnal Emerg Med. 2012. 